\title{
Crystal Structure of a 2-Biphenyl-2-yl-1,1,3,4-tetraphenyl-1H-silole
}

\author{
Youngjin KANG, ${ }^{* \dagger}$ Ki-Min PARK, ${ }^{* *}$ and Soon-Ki KwON** \\ *Division of Science Education, Kangwon National University, Chuncheon, 200 - 701, Korea \\ **Research Institute of Natural Sciences, School of Applied Chemical Engineering \& Engineering Research \\ Institute, Gyeongsang National University, Chinju 660 - 701, Korea
}

\begin{abstract}
Crystals of silole ( silole $=$ silacyclopentadiene) analogue, 2-biphenyl-2-yl-1,1,3,4-tetraphenyl-1 $H$-silole, were grown from a dichloromethane/hexane solution and the crystal structure was determined. The crystal belongs to the monoclinic system with space group of $P 2_{1} / c$. There is an intramolecular $\pi-\pi$ interaction in an asymmetric unit, and the shortest distance between one of the phenyl rings in the biphenyl and the phenyl ring at the 3-position of silole is about $3.643 \AA$. This silole can be used as a potential building block for generating linear polymers containing the silole moiety owing to the labile nature of the proton attached at the 2-position.
\end{abstract}

(Received March 12, 2004; Accepted April 26, 2004; Published on Web June 17, 2004)

Of a variety of the organosilicon compounds, silole (silole = silacyclopentadiene) derivatives have attracted much attention in material science owing to their various applications to conductors, OLEDs (organic light-emitting diodes) and nonlinear optics. ${ }^{1}$ By introducing various functional groups at the 2 and 5 positions, siloles have been shown to have unique electronic properties and novel molecular structures. Based on previous reports, a series of siloles with the same functional group at the 2 and 5 positions were prepared readily by a Pdmediated one-step reaction via intramolecular reductive cyclization when employing bis(alkyl or arylethynyl)silanes. ${ }^{2}$ Although the crystal structures and optical properties of 2,5disubstituted siloles, containing the same group at the 2 and 5 positions, have been well described in the past several years, the number of analyzed structures of silole with different groups at those positions, such as 2-alkyl-5-arylsilole, 2-halo-5-alky(or aryl)silole and 2 -alky(or aryl)-5H-silole etc., still remains limited. In our ongoing development of silole derivatives, ${ }^{3}$ we found that mono-substituted siloles at the 2 or 5 position were formed as major components, depending on reaction conditions. Herein, we describe the structure of mono-substituted silole, 2biphenyl-2-yl-1,1,3,4-tetraphenyl-1H-silole (Fig. 1), based on an X-ray crystal analysis.

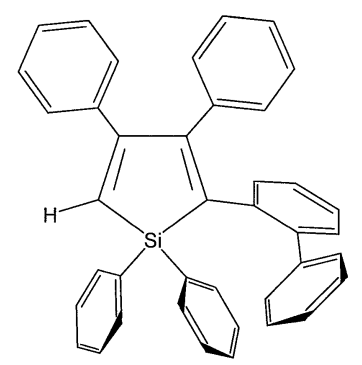

Fig. 1 Chemical structure.

To whom correspondence should be addressed.

E-mail : kangy@kangwon.ac.kr, skwon@gsnu.ac.kr
2-Biphenyl-2-yl-1,1,3,4-tetraphenyl- $1 H$-silole was prepared by a slight modification of the synthetic methodology reported by Tamao's group. ${ }^{2}$ Pale-yellow crystals suitable for X-ray crystallography were grown by slow evaporation from dichloromethane/hexane at room temperature. The crystal and experimental data are depicted in Table 1. The structure was solved by a direct method. The bond length of $\mathrm{C} 2-\mathrm{C} 18$ is $1.498(6) \AA$, which is slightly longer than those of other 2,5diarylsiloles, such as 1,1-dimethyl-2,5-bis( $p$-nitrophenyl)-3,4diphenylsilole $\quad(1.475(2) \AA), \quad$ 1,1-dimethyl-2,5-bis $(p$ -

Table 1 Crystal and experimental data

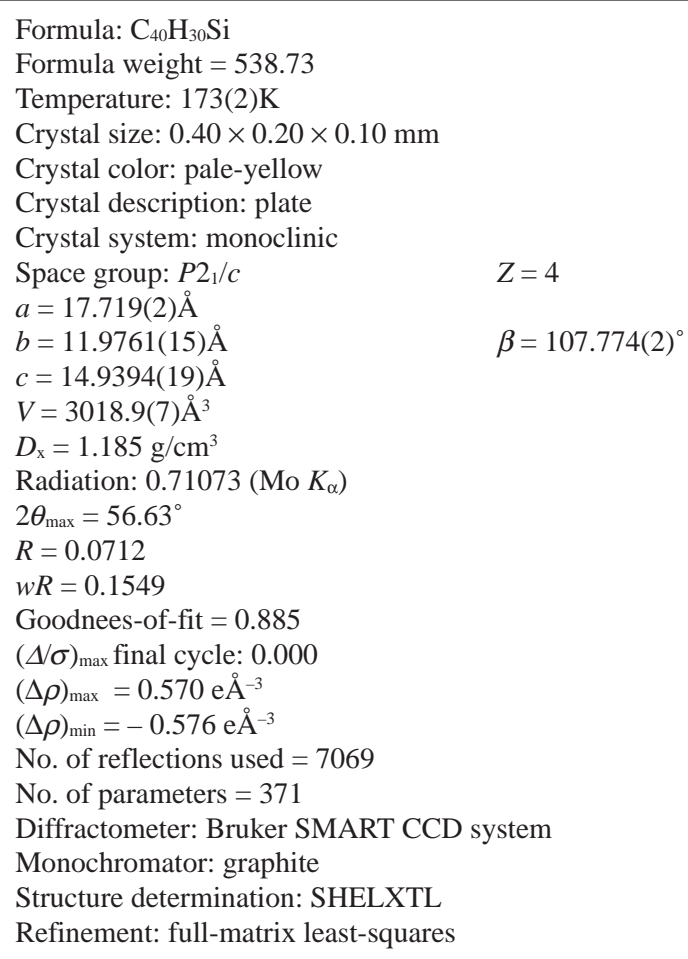

Formula weight $=538.73$

Temperature: $173(2) \mathrm{K}$

Crystal size: $0.40 \times 0.20 \times 0.10 \mathrm{~mm}$

Crystal system: monoclinic

Space group: $P 2{ }_{1} / c$

$a=17.719(2) \AA$

$D_{\mathrm{x}}=1.185 \mathrm{~g} / \mathrm{cm}^{3}$

$2 \theta_{\max }=56.63^{\circ}$

$R=0.0712$

$w R=0.1549$

Goodnees-of-fit $=0.885$

$(\alpha)$ final cycle: 0.000

No. of reflections used $=7069$

No. of parameters $=371$

Monochromator: graphite

Refinement: full-matrix least-squares 


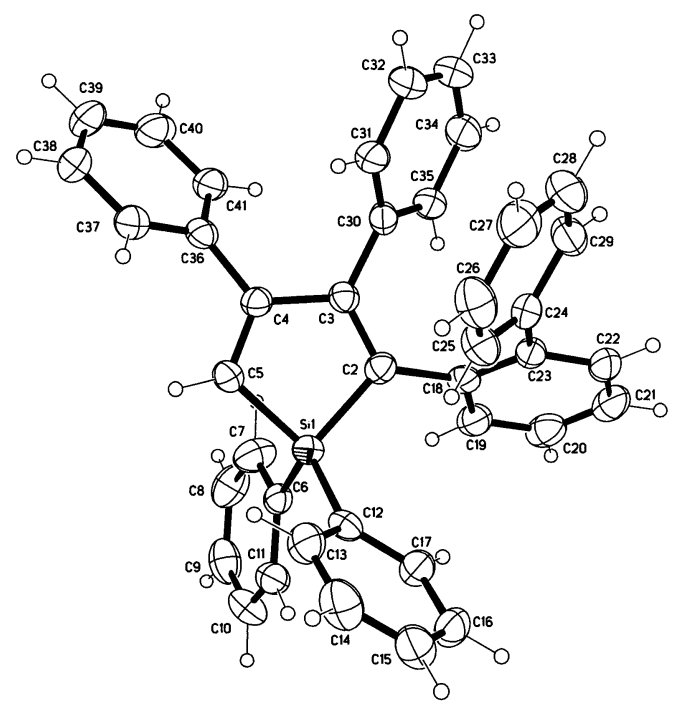

Fig. 2 Crystal structure of the titled compound with the atomicnumbering scheme for Table 2. Displacement ellipsoids are drawn at the $50 \%$ probability level.

Table 2 Atomic coordinates $\left(\times 10^{4}\right)$ and equivalent isotropic displacement parameters $\left(\AA^{2} \times 10^{3}\right)$

\begin{tabular}{|c|c|c|c|c|}
\hline Atom & $x$ & $y$ & $z$ & $U(\mathrm{eq})$ \\
\hline $\mathrm{Si}(1)$ & $1324(1)$ & $2110(1)$ & $1112(1)$ & $31(1)$ \\
\hline $\mathrm{C}(2)$ & 2034(3) & $1798(3)$ & $441(3)$ & $30(1)$ \\
\hline$C(3)$ & $2609(2)$ & $2591(3)$ & $647(3)$ & $28(1)$ \\
\hline $\mathrm{C}(4)$ & $2508(2)$ & $3496(3)$ & $1296(3)$ & $29(1)$ \\
\hline $\mathrm{C}(5)$ & 1929(3) & $3321(3)$ & $1710(3)$ & $33(1)$ \\
\hline$C(6)$ & $341(3)$ & $2628(3)$ & $352(3)$ & $31(1)$ \\
\hline $\mathrm{C}(7)$ & $314(3)$ & $3496(4)$ & $-269(4)$ & $49(1)$ \\
\hline $\mathrm{C}(8)$ & $-386(3)$ & $3961(4)$ & $-808(4)$ & $52(1)$ \\
\hline $\mathrm{C}(9)$ & $-1104(3)$ & $3577(4)$ & $-711(3)$ & $50(1)$ \\
\hline$C(10)$ & $-1096(3)$ & $2705(4)$ & $-118(3)$ & $46(1)$ \\
\hline$C(11)$ & $-388(3)$ & $2247(4)$ & $425(3)$ & $37(1)$ \\
\hline $\mathrm{C}(12)$ & $1194(2)$ & $901(4)$ & $1832(3)$ & $29(1)$ \\
\hline$C(13)$ & $1402(3)$ & $922(4)$ & $2810(3)$ & $42(1)$ \\
\hline $\mathrm{C}(14)$ & $1321(3)$ & $-32(5)$ & $3318(4)$ & $51(1)$ \\
\hline$C(15)$ & $1029(3)$ & $-994(4)$ & $2859(4)$ & $46(1)$ \\
\hline$C(16)$ & $815(3)$ & $-1037(4)$ & $1896(4)$ & $43(1)$ \\
\hline $\mathrm{C}(17)$ & 904(3) & $-106(4)$ & $1400(3)$ & $36(1)$ \\
\hline$C(18)$ & $1986(3)$ & $819(4)$ & $-198(3)$ & $29(1)$ \\
\hline $\mathrm{C}(19)$ & 1302(3) & $737(4)$ & $-972(3)$ & $39(1)$ \\
\hline $\mathrm{C}(20)$ & $1213(3)$ & $-123(4)$ & $-1618(4)$ & $47(1)$ \\
\hline $\mathrm{C}(21)$ & $1801(4)$ & $-924(4)$ & $-1492(4)$ & $50(2)$ \\
\hline $\mathrm{C}(22)$ & $2468(3)$ & $-870(4)$ & $-711(4)$ & $45(1)$ \\
\hline$C(23)$ & $2570(3)$ & $-23(4)$ & $-45(3)$ & $32(1)$ \\
\hline $\mathrm{C}(24)$ & $3281(3)$ & $-49(4)$ & $796(4)$ & $38(1)$ \\
\hline$C(25)$ & $3221(3)$ & $54(4)$ & $1700(4)$ & $44(1)$ \\
\hline $\mathrm{C}(26)$ & 3893(3) & $17(4)$ & $2484(4)$ & $52(1)$ \\
\hline $\mathrm{C}(27)$ & $4632(3)$ & $-111(4)$ & $2377(4)$ & $57(2)$ \\
\hline $\mathrm{C}(28)$ & $4701(3)$ & $-223(4)$ & $1479(4)$ & $52(2)$ \\
\hline$C(29)$ & $4035(3)$ & $-192(4)$ & $691(4)$ & $43(1)$ \\
\hline $\mathrm{C}(30)$ & $3318(3)$ & $2594(3)$ & $296(3)$ & $29(1)$ \\
\hline$C(31)$ & $4065(3)$ & $2773(4)$ & 914(4) & $40(1)$ \\
\hline $\mathrm{C}(32)$ & $4730(3)$ & $2790(4)$ & $620(4)$ & $46(1)$ \\
\hline$C(33)$ & $4639(3)$ & $2626(4)$ & $-333(4)$ & $58(2)$ \\
\hline$C(34)$ & $3903(3)$ & $2441(4)$ & $-965(4)$ & $51(2)$ \\
\hline$C(35)$ & $3244(3)$ & $2417(4)$ & $-644(3)$ & $35(1)$ \\
\hline $\mathrm{C}(36)$ & $2935(2)$ & $4578(4)$ & $1403(3)$ & $31(1)$ \\
\hline$C(37)$ & $3142(3)$ & $5151(4)$ & $2272(3)$ & $38(1)$ \\
\hline$C(38)$ & $3445(3)$ & $6226(4)$ & $2342(4)$ & $48(1)$ \\
\hline $\mathrm{C}(39)$ & $3557(3)$ & $6754(4)$ & $1560(4)$ & $52(2)$ \\
\hline $\mathrm{C}(40)$ & $3369(3)$ & $6191(4)$ & $718(4)$ & $51(1)$ \\
\hline $\mathrm{C}(41)$ & $3068(3)$ & $5120(4)$ & $638(4)$ & $41(1)$ \\
\hline
\end{tabular}

$U_{\mathrm{eq}}=1 / 3 \Sigma_{i} \Sigma_{j} U_{i j} a_{i}^{*} a_{j}^{*}\left(\boldsymbol{a}_{i} \cdot \boldsymbol{a}_{j}\right)$.
Table 3 Selected bond lengths $(\AA)$ and angles $\left({ }^{\circ}\right)$

\begin{tabular}{lcll}
\hline $\mathrm{Sil}-\mathrm{Cl} 2$ & $1.860(4)$ & $\mathrm{Si1}-\mathrm{C} 5$ & $1.862(4)$ \\
$\mathrm{Si} 1-\mathrm{C} 2$ & $1.870(4)$ & $\mathrm{Sil}-\mathrm{C} 6$ & $1.872(6)$ \\
$\mathrm{C} 4-\mathrm{C} 5$ & $1.365(5)$ & $\mathrm{C} 2-\mathrm{C} 18$ & $1.498(6)$ \\
$\mathrm{C} 3-\mathrm{C} 4$ & $1.501(6)$ & $\mathrm{C} 2-\mathrm{C} 3$ & $1.357(6)$ \\
& & & \\
$\mathrm{C} 2-\mathrm{Si} 1-\mathrm{C} 5$ & $91.71(18)$ & $\mathrm{C} 6-\mathrm{Si}-\mathrm{C} 12$ & $110.60(19)$ \\
$\mathrm{Si}-\mathrm{C} 5-\mathrm{C} 4$ & $107.7(3)$ & $\mathrm{Sil}-\mathrm{C} 2-\mathrm{C} 3$ & $108.6(3)$ \\
$\mathrm{Si} 1-\mathrm{C} 2-\mathrm{C} 18$ & $125.5(3)$ & $\mathrm{C} 2-\mathrm{C} 3-\mathrm{C} 4$ & $115.0(4)$ \\
\hline
\end{tabular}

methoxyphenyl)-3,4-diphenylsilole (1.480(2) ̊), 1,1-dimethyl2,5-bis(p-methylphenyl)-3,4-diphenylsilole $(1.479(2) \AA)$ and 1,1-dimethyl-2,5-bis( $p$-trifluoromethylophenyl)-3,4diphenylsilole $(1.473(3) \AA)^{3}{ }^{3} \quad$ Notably, the shortest distance (C24-C30) between one of the phenyl rings in the biphenyl and the phenyl ring at the 3-position of silole is about 3.257 $\AA$, indicating that there is an intramolecular $\pi$ - $\pi$ interaction in an asymmetric unit, as shown in Fig. 2. These two phenyl rings (C24-C29 and C30-C35) are almost parallel with a dihedral angle of $12.37(8)^{\circ}$, while the two phenyl rings between biphenyl (C18-C23 and C24-C29) are significantly tilted by an angle of $49.75(15)^{\circ}$. This result can be explained by a steric repulsion between $\mathrm{C} 18$ and $\mathrm{H} 25$ attached at $\mathrm{C} 25$ (atropisomerism). The $\mathrm{H}$-atoms were calculated geometrically, $0.95 \AA$ from the corresponding atoms, and refined using a riding model. The atomic coordinates and the selected geometric parameters are presented in Tables 2 and 3, respectively. There are no significant intermolecular interactions (e.g. $\pi$ - $\pi$ stacking) in the crystal packing. The large positive $\left(0.57 \mathrm{e}^{-3}\right)$ and negative $\left(-0.58 \mathrm{e}^{-3}\right)$ difference Fourier peaks are located at short distances from C32 (0.69 $\AA$ ) and H31A (1.37 $\AA$ ), respectively; these peaks can be attributed to ghosts.

\section{Acknowledgements}

The support of Kangwon National University is gratefully acknowledged.

\section{References}

1. (a) K. Tamao, S. Yamaguchi, and M. Shiro, J. Am. Chem. Soc., 1994, 116, 11715. (b) M. Uchida, T. Izumizawa, T. Nakano, S. Yamaguchi, and K. Tamao, K. Furukawa, Chem. Mater., 2001, 13, 2680. (c) H. Y. Chen, W. Y. Lam, J. D. Luo, Y. L. Ho, B. Z. Tang, D. B. Zhu, M. Wong, and H. S. Kwok, Appl. Phys. Lett., 2002, 81, 574.

2. S. Yamaguchi, T. Endo, M. Uchida, T. Izumizawa, K. Furukawa, and K. Tamao, Chem. Eur. J., 2000, 6, 1683.

3. Y. Kang, J. Park, J. Heo, Y. Kim, and S. K. Kwon, Nonlinear Opt. Phys. Mater., 2004, submitted. 